\title{
Effect of light filters on reading speed in normal and low vision due to
}

age-related macular degeneration

Frank Eperjesi ${ }^{1,2}$, Colin W. Fowler ${ }^{1}$, Bruce J. W. Evans ${ }^{3,4}$

1. Neurosciences Research Institute, School of Life and Health Sciences, Aston University, Aston Triangle, Birmingham, B4 7ET, UK.

2. Low Vision Centre, Birmingham Focus on Blindness, 62 Woodville Road, Harborne, Birmingham, B17 9AX, UK.

3. The Institute of Optometry, 56-62 Newington Causeway, London, SE1 6DS, UK.

4. Department of Optometry and Visual Science, City University, Northampton Square, London EC1V OHB, UK.

Corresponding address: F Eperjesi, Neurosciences Research Institute, School of Life and Health Sciences, Aston University, Birmingham, B4 7ET, UK. E-mail address: f.eperjesi@aston.ac.uk 


\section{Abstract}

Purpose: To investigate the effects of light filters on reading speed in normal and low vision due to age-related macular degeneration (AMD).

Methods: Reading speed was determined for twelve subjects with normal vision and twelve subjects with non-exudative AMD using stationary lowercase nonsensical print in Times Roman font and four light filters; a yellow Corning Photochromic Filter (CPF) 450, a grey neural density (ND) filter, an individual filter obtained using the Intuitive Colorimeter ${ }^{\circledR}$ and a clear filter.

Results: There was no statistically significant light filter effect on reading speed for the normal subjects. The AMD group demonstrated a statistically significant $5 \%$ average improvement in reading speed with the CPF450 compared to the other filters although some AMD subjects had improvements of 10 to $15 \%$. Conclusions: Light filters obtained using the Intuitive Colorimeter® performed poorly when compared to the CPF450, ND and clear filters for both the study groups. For the AMD group, average reading speed was statistically greater with the CPF450 than the other filters, however it is questionable whether the improvement (5\%) would be clinically significant. As some of the subjects with AMD had greater improvements with the CPF450 we advocate clinical assessment of light filters using existing protocols on an individual basis.

Keywords: age-related macular degeneration, light filters, CPF450, Intuitive Colorimeter® 


\section{Introduction}

People with low vision sometimes use light filters in the form of tinted spectacle lenses in an attempt to improve visual function, visual comfort and orientation and mobility. Numerous types are available, in many different colours, and they can be used for in door and out door purposes. Several studies have assessed the effect of short-wavelength absorbing filters (often termed blue-blockers) on visual performance in low vision. Some have reported a subjective preference for yellow and orange filters (Hoeft and Hughes, 1981; Morrissette et al., 1984; Maino and McMahon, 1986; Provines et al., 1997) while others have demonstrated objective improvements in visual performance (Hellinger, 1983; Lynch and Brilliant, 1984; Tupper et al., 1985; Maino and McMahon, 1986; Zigman, 1990; Frennesson and Nilsson, 1993; Rosenblum et al. (2000); Wolffsohn et al., 2002). Much of the objective data collected involves clinical measures that correlate poorly with subjective quality of life e.g. visual acuity, rather than measures that are better predictors of quality of life, such as contrast sensitivity (Marron and Bailey, 1982; Leguire and Suh, 1993; Dickinson, 1998; Rosenblum et al., 2000) and reading speed (Hazel et al., 2000; McClure et al, 2000). See Eperjesi et al. (2002) for a review of the use of light filters in low vision.

The most common cause of permanent vision loss in the developed world is AMD (Elliott et al., 1997) and one of the chief complaints expressed by people with this condition is difficulty with reading at near (Hazel et al 2000). Although light filters have been shown to have no effect on reading speed for stationary print in cataract (Bailey et al., 1978) or reading speed for moving words 
presented on a TV screen for central field loss (Legge and Rubin, 1986) to our knowledge the effect of light filters on reading speed for stationary printed text has not been investigated for AMD subjects.

The purpose of this study was to compare the effects of three light filters [a commercially available yellow glass photochromic filter (CPF450); a ND filter; a plastic, surface tinted filter (derived using the Intuitive Colorimeter ${ }^{\circledR}$ )] with a clear filter on reading speed for subjects with normal and low vision due to nonexudative AMD. We were particularly interested in comparing the performance of CPF450 with the Intuitive Colorimeter ${ }^{\circledR}$ light filter for the AMD group, because the CPF450 is generic and we wondered whether reading speed might be improved with a light filter derived for each subject on an individual basis.

\section{Method}

Subjects

Twenty-four subjects took part in the study. Twelve (four males and eight females) had non-exudative AMD with central field loss (mean age 82.4 years, range 73 to 87 years, standard deviation (SD) 4 years). Near word reading acuity ranged from log MAR 0.30 to 1.00 , with a mean of 0.60 . The other participants (six males and six females) were normally sighted (mean age 69.3 years, range 60 to 74 years, SD 4) without any ocular disease. Near wording reading acuity for this group ranged from log MAR 0.10 to 0.30 , with a mean of 0.25 . None of the participants were pseudophakic and all had crystalline lens opacities graded as one or less on the Lens Opacity Classification System 
(Chylack et al., 1988). People with a near wording reading acuity of poorer that logMAR 1.00 (approximately equivalent to large print) or with crystalline opacities greater than grade one on the Lens Opacity Classification System or who were undergoing ophthalmological treatment were excluded from the study.

\section{Materials}

Light filters used in this study were: CPF 450 in the form of a plano, glass photochromic clip-on, absorbing below $450 \mathrm{~nm}$, with 68\% total light transmission (TLT) in the lightened state (Norville Group, Paul Street, Gloucester, GL1 4NY, UK); a plastic, surface tinted filter determined for each subject individually using the Intuitive Colorimeter®) (Cerium Visual Technologies, Cerium Technology Park, Appledore Road, Tenterden, Kent, TN30 7DE, UK); a ND filter (50\% TLT) clip-on; a clear filter (100\% TLT) clip-on. The CPF range is marketed to improve visual function, visual comfort and mobility performance for people with low vision. CPF450 (light yellow), the palest of the CPF range, is designed for indoor use and is claimed by the manufacturers to enhance reading in AMD.

TLT was determined for each filter in the following way. A Chauvin Arnoux CA 810 Luxmeter (Chauvin Arnoux, 853 Plymouth Road, Slough, SL1 4LP, UK) was used to measure the illuminance (in lux) produced by a $11 \mathrm{~W}$ compact fluorescent lamp positioned $25 \mathrm{~cm}$ directly above the lux-meter sensor. Each light filter was placed in turn on the lux-meter sensor. The illuminance value with and without each filter in place was noted and the difference calculated as 
a percentage to provide the TLT. Ambient illuminance remained constant throughout this procedure.

The Intuitive Colorimeter ${ }^{\circledR}$ was developed by Wilkins to assess those people who suffer with pattern-glare (Wilkins et al., 1992). This has been described as the occurrence of perceptual distortions due to sensitivity to stripy patterns including the pattern formed by printed text at near. These perceptual distortions often take the form of coloured fringes and ghost images around letters, moving letters or words, and straight lines of words appearing wavy. The Intuitive Colorimeter® is a 'black box' table mounted instrument that contains red, green and blue filters for mixing coloured light that can be used with an internal beam of collimated white light for the independent manipulation of hue, saturation and brightness. It has a viewing port through which observers can read stationary printed text mounted on a flat surface approximately parallel to the plane of the face at a distance of $25 \mathrm{~cm}$.

The Rate of Reading Test ${ }^{\circledR}$ (RRT) (IOO Marketing Ltd., 56-62 Newington Causeway, London, SE1 6DS, UK) is a simple reading test that minimises the linguistic and semantic aspects of reading and maximises the visual aspects. Subjects are required to read aloud a passage of 15 randomly ordered words (see, the, look, dog, and, not, is, you, come, up, play, to, my, for, cat) as rapidly as possible. The same words are used in each line, but in a different order. The test is designed to compare an individual's performance under one set of conditions with that under another and has been shown to be a reliable and valid indicator of reading speed when assessing the effects of a chromatic 
optometric intervention for normally sighted children 8 to 11 years old (Wilkins et al., 1996). Reading speed is considered to be a good measure of reading performance because most errors tend to decrease the speed either by reducing the number of words correctly read or by increasing the time taken to read the passage (Wilkins et al., 1996).

The RRT words were typed into a word processor and printed in Arial font sizes ranging from four to 18-point. Twelve different paragraphs in each font size, with 10 lines and 15 words per line, resulting in a total of 150 words per block were used to determine reading speed in stage 1 of the study. See figure 1 for an example of a RRT test block. Five different versions of the Bailey-Lovie near word reading acuity card (a series of unrelated words printed in Times Roman print, ranging in size from N80 to N2, spanning a 1.60 log unit size range (two, three or six words per line) were used to determine reading speed in stage 2.

To our knowledge neither the Intuitive Colorimeter ${ }^{\circledR}$ nor the RRT have been used on subjects with central field loss and therefore reliability and validity data are not available.

Figure 1 about here Procedure

All participants gave informed consent to take part in the study, which was approved by the Institutional Human Ethics Committee. The study comprised two stages; stage 1 in which the Intuitive Colorimeter® was used to determine an optimum filter (in terms of reading speed) for each subject individually and 
stage 2 in which reading speed was measured for all participants using each of the four study filters.

\section{STAGE 1}

The following procedure was used in to obtain an optimum filter using the Intuitive Colorimeter ${ }^{\circledR}$ for each participant. Testing was conducted with room lights off (35 lux ambient illuminance, as measured on horizontal desk surface $0.74 \mathrm{~m}$ above the floor). A block of RRT text (printed right and left justified in lower case Arial font with single line spacing) was chosen for each subject to be 0.10 logMAR units greater than threshold binocular reading acuity as measured with the Bailey-Lovie word reading card at the habitual reading distance. Our previous experience has indicated that testing at reading acuity threshold results in visual fatigue and visual discomfort that often leads to the subject having difficulty completing the test session. This block of text was placed into the Intuitive Colorimeter®. Prior to the start of the testing session, participants were shown large print (N40) versions of the RRT words and asked to read them out loud to encourage familiarity. They were informed that the test blocks contained text comprised solely of these words, each line had a different arrangement of the same words, there were no contextual clues and their reading 'would not make sense'. For each subject the same font size was used in each test block, but font size varied from subject to subject according to their near threshold word reading acuity. Participants were asked to read out loud from the blocks, as accurately and as quickly as possible starting at the top left hand corner of each block. Subjects were encouraged to guess or pass on 
words they were unsure about. Blocks of test print were presented in the same order for all subjects.

Each subject was asked to read one block of 150 words for each hue generated by the Intuitive Colorimeter $\AA^{\circledR}$. The illuminance inside the Intuitive Colorimeter was 120 lux, which along with saturation remained constant for all presentations. Subjects were advised to read at their habitual working distance and this was monitored by observation for consistency (inter-subject range from 25 to $35 \mathrm{~cm}$ ). The test session was tape-recorded and later analysed to calculate the optimum hue in terms of reading speed in correct words per minute (cwpm) for each subject. Twelve hues were presented consecutively (same order for each subject) each being separated by 30 degrees on the hue wheel, starting at zero and finishing at 330 degrees, a standardized sequence recommended in the manual that accompanies the Intuitive Colorimeter ${ }^{\circledR}$ (Wilkins, 1993). In order to allow some practice, each participant was asked to read one test block that was illuminated by an internal fluorescent tube only (no coloured filter in place). This was repeated at the end of the test session for each participant and the average 'before' and 'after' reading speed values for each group were compared in order to detect any learning and fatigue effects. The longest test session lasted 30 minutes. For each subject the hue that resulted in the highest reading rate was matched with a combination of tinted trial lenses that accompany the Intuitive Colorimeter ${ }^{\circledR}$. All optimum hues were matched with one or a combination of two trial lenses and these were used in stage 2 of the study as the Intuitive Colorimeter® filter. 


\section{STAGE 2}

Participants were seated at a desk illuminated by an $11 \mathrm{~W}$ cool white compact fluorescent lamp placed at $25 \mathrm{~cm}$ above the desk surface. Testing was conducted in a well-lit room with ceiling mounted fluorescent lamps. Total illuminance at the horizontal desk surface $0.74 \mathrm{~m}$ above the floor was 3830 lux (combined ambient and localised lighting). Each subject was given the same instructions; none were aware of the possible benefits to reading speed of any of the filters and all were tested binocularly. A Bailey-Lovie near word reading acuity card was placed flat on the desk and subjects were allowed to read at their habitual working distance with their current reading spectacles. Subjects were advised not to move or tilt the card during the test session and not to move closer when attempting to read small or difficult words. They were encouraged to guess or pass on words they were unsure about. All subjects were informed that the words had no contextual clues and therefore their reading would not make sense. Subjects were also advised that they would be tape-recorded and the tape would be analysed later to determine reading speed. Participants were asked to read out loud from the card, as accurately and as quickly as possible starting at the top of the card and reading down until the words were too small to see. All subjects could complete at least one line that contained six words. The end point was reached when more than fifty percent of the words on one line were read incorrectly (i.e. four or more). A similar procedure was used by Bailey et al (1978) to investigate the effects of light filters on reading speed with real and simulated cataract. 
The CPF450, ND and clear filters were mounted as plano clip-ons and attached directly to subjects' own spectacles. The CPF450 was used in the fully lightened state (68\% TLT). Intuitive Colorimeter ${ }^{\circledR}$ filters were hand held by each subject in front of each eye at the same time, close up to their own spectacles. During testing subjects were given two minutes to adapt to each filter while maintaining fixation on the word reading card. All word reading cards and light filters were randomly selected in order to prevent the effects of learning and fatigue. No test session lasted longer than 15 minutes.

\section{Results}

See tables 1 and 2 for reading speeds for normal and AMD subjects from stage 1 of the study using hues generated by the Intuitive Colorimeter ${ }^{\circledR}$ and tables 3 and 4 for reading speeds obtained for each group using light filters from stage 2.

Table 1, 2, 3 and 4 here

Hue versus reading speed

We wondered whether there was a relationship between reading speed and optimum Intuitive Colorimeter ${ }^{\circledR}$ hue (the hue that resulted in the greatest reading speed for each individual in stage 1) for either group. Therefore, we carried out a main effects ANOVA using StatSoft, Inc. (2001), STATISTICA (data analysis software system), version 6 (www.statsoft.com) with reading speed as the dependent variable and subjects and hue as categorical factors. For the normal subjects $F, 1.39, p=0.178$, df 12 and for the AMD subjects $F$, 
$0.513, p=0.903, \mathrm{df} 12$. These results indicate that there was no statistically significant relationship between reading speed and Intuitive Colorimeter® hue. When 'before' and 'after' reading speeds (internal fluorescent illumination only) were compared using Student's t test, no statistically significant difference was found for the normal $(t=-0.017, p=0.868)$ or AMD subjects $(t=-0.343, p=0.735)$. This suggests that the reading speed results are unlikely to have been confounded by learning and fatigue effects.

\section{$T L T$ and reading speed}

In order to determine whether there was a relationship between TLT of the optimum Intuitive Colorimeter ${ }^{\circledR}$ filters (stage 2) and reading speed (see tables 3 and 4) we calculated Pearson's 'r' for each subject group with reading speed as the dependent variable. For the normal group $r=0.51$ and for the AMD group $r$ $=0.19$, neither was significant at the $5 \%$ level. These results indicate that there was no statistically significant relationship between reading speed and optimum Intuitive Colorimeter® filter TLT.

ANOVA and post-hoc analyses

Reading speeds obtained in stage 2 were compared for each subject group and each filter using a three-factor repeated measures ANOVA procedure with the presence of AMD and filter type as major factors and reading speed as a sub-plot split factor (Armstrong et al., 2002). A significant filter effect on reading speed for the AMD group was found $(F, 3.62, p=0.023$, df 0,33$)$ but not for the normal group ( $F, 1.27, p=0.300$, df 0,33$)$. Data from the AMD group were further analysed using the Duncan New Multiple Range post-hoc test and significant relationships between the CPF450 and ND filter ( $p \leq 0.05)$, and the 
CPF450 and the Intuitive Colorimeter ${ }^{\circledR}$ filter $(p \leq 0.05)$ were detected. Analysis by observation of the average values for each filter for the AMD group indicated that the CPF450 produced a greater reading speed than the ND and Intuitive Colorimeter® filters.

Normalised reading speed

Normalised reading speed values for each light filter and each group were calculated by dividing the average reading speed for each of the light filters by the average reading speed obtained with the clear filter. For the normal group, all the light filters produced an average reading speed that was poorer than that produced by the clear filter. For the AMD group only the CPF450 produced a greater normalised average reading speed than the clear filter. See figure 2.

Figure 2 here

\section{Discussion}

CPF450 is a commercially available yellow glass photochromic light filter that has been promoted by the manufacturers as a reading aid in low vision and in particular for people with AMD. We consider it to be an 'off the shelf generic' device and were interested in comparing reading performance using the CPF450 with ND, individually determined (Intuitive Colorimeter $\left.{ }^{\circledR}\right)$, and clear light filters in normal and low vision due to AMD with reading speed as the outcome measure. 
We found that there was no statistically significant relationship between Intuitive Colorimeter ${ }^{\circledR}$ hue and reading speed for either group; in other words when saturation and brightness were kept constant there was no hue effect on reading speed. The Intuitive Colorimeter ${ }^{\circledR}$ can be used to obtain a light filter on an individual basis but, in general, these filters performed poorly compared to the other study filters. The poor performance of the Intuitive Colorimeter® light filters could be due to three confounding factors. Firstly, this was the only filter that was hand held (the others were all spectacle mounted) however, none of the subjects complained and none were observed to have any difficulties holding the filters. In hindsight, this confounding factor could have been avoided by glazing a clip-on frame with plano lenses carrying the optimum Intuitive Colorimeter® tint for each subject. Secondly, several of the individually determined Intuitive Colorimeter® filters had low TLT values compared to the other study filters (see table 3 and 4 ). For example, the Intuitive Colorimeter® filters for normal subject KG and AMD subject MH had a TLT of $15 \%$ and $16 \%$ reducing task illumination from 3830 lux to 613 and 575 lux respectively. Surprisingly, we did not find a statistically significant correlation between Intuitive Colorimeter® filter TLT and reading speed for either group, and therefore variable TLT is unlikely to have been a confounding factor. This finding is in contrast to that reported by Bowers et al (2001) who found that the majority of their AMD sufferers required a task illuminance of 2000 lux to maximise reading performance. Thirdly, the eye has substantial longitudinal chromatic aberration and short-wavelength light focuses anteriorly compared to light of longer wavelength (Bedford and Wyszecki, 1957). It has been suggested that for an eye accommodating by 3.00DS, short wavelengths (380 
$\mathrm{nm}$ ) could result in approximately $-2.00 \mathrm{DS}$ of blur and long wavelengths (780 $\mathrm{nm}$ ) approximately $+0.60 \mathrm{DS}$ of blur at the retina compared to medium wavelengths (Bennett and Rabbetts, 1989). Some of the Intuitive Colorimeter ${ }^{\circledR}$ filters used by subjects in both our study groups were predominantly red or blue in colour and as we did not compensate for chromatic aberration using spherical lenses, retinal blur may have resulted in lower reading speeds with these filters. However, in stage 1 of the study several subjects in each group had greater reading speeds with blue or red than with yellow hue and this broadly agrees with Campbell and Gubisch (1967) who found very little effect of chromatic aberration on visual acuity. We consider it unlikely that retinal blur due to chromatic aberration confounded the results from stage 2 .

Abramov and Gordon (1977) found that the peripheral retina of normal observers had an enhanced sensitivity to short wavelengths and as people with central scotoma must rely more on peripheral vision they may use paramacular and peripheral retina to read, it could advantageous for them to read with blue hues. Our results, indicating no benefit from blue light filters and those of Legge and Rubin (1986) who reported that, for observers with central field loss (compensated for chromatic aberration), there was no difference in reading performance between blue and red filters, would suggest that this is not the case.

Post hoc analysis of an ANOVA revealed a significant improvement in average reading speed with the CPF450 when compared to the other filters for the AMD group. The average improvement was $5.51 \mathrm{cwpm}$ (approximately 5\%) but when 
taking into account the high intra-group reading speed variability with the CPF450 (almost $\mathrm{x} 12$ between the slowest and fastest readers) it is questionable whether a $5 \%$ improvement in reading speed is likely to be of clinical significance. However, when reviewing the individual data one AMD subject had a 16 cwpm increase in reading speed with the CPF 450, which would equate to almost 1000 extra words per hour or three A4 pages of N14 print. In the clinical setting we support protocols proposed by Pensyl (1993) and Gormezano and Stelmack (2000) for individual testing prior to the prescription of selective absorption filters. The effect of a range of filters, such as yellow and grey tints, can be objectively and subjectively be compared using high contrast visual acuity and contrast sensitivity charts under various types of illumination in and out doors along with assessment of near point preference using a variety of printed materials.

An anonymous reviewer of an earlier version of this paper noted that the that there was almost as much range in reading speed for the normally sighted subjects as there was for those with AMD, and that one normally sighted subject (GM-table 3) read at a speed that was lower than all but one of the subjects with AMD. We are unsure as to the reason for this but it is possible that some participants were concerned about making errors when reading out loud in an experimental testing situation and read more carefully and therefore more slowly that they would normally under non-test circumstances. We asked our subjects to read out loud and used reading speed as an outcome measure because our main aim was to compare reading performance in a research setting between four light filters and reading speed has been shown to be a 
sensitive psychophysical measure of performance with different visual interventions (Wilkins et al, 1996) that relates well to subjective quality of life (Hazel et al., 2000; McClure et al, 2000).

It would be useful to know whether fixed tint plastic lenses perform as well as the CPF range. For future studies comparing the effects light filters as well as light filter evaluation in the clinical setting we suggest the use of reading comprehension as a performance indicator using a near point test such as the MNRead cards (reading acuity/speed cards that use sentences of equivalent length and level of comprehension) (Ahn et al, 1995). This would allow silent reading and the use of accuracy of comprehension as a performance measure which although is a poor psychophysical measure (Legge et al, 1989) has been shown to relate reasonably well to real world reading (Leat et al, 1992). Furthermore, it is possible that light filters improve visual comfort and that this may lead to increased duration of reading which could be more useful than increased reading speed. Therefore, reading productivity (the product of reading speed in cwpm and reading duration in minutes) or how much a person can accomplish in a given period of time (Goodrich et al, 2000) could be used as a performance indicator instead of single measure of reading speed.

In conclusion, participants with normal vision did not benefit when reading with light filters compared to a clear filter. Light filters obtained using the Intuitive Colorimeter ${ }^{\circledR}$ performed poorly when compared to the other study filters and are unlikely to be of benefit for people with AMD. CPF450 was the only light filter to produce a statistically significant improvement in reading speed for our 
sample of AMD subjects. The average improvement was $5 \%$ although some individuals demonstrated a 10 to $15 \%$ improvement and we therefore advocate the use of published protocols for the prescription of light filters whereby each person undergoes a light filter evaluation on an individual basis.

\section{Declaration of commercial interest}

None of the authors have any commercial interest in the instrumentation or light filters discussed in this paper.

\section{Acknowledgements}

We would like to thank Dr RA Armstrong for assistance with the statistical analyses and Dr JS Wolffsohn for assistance with figure 2.

\section{References}

Abramov, I. and Gordon, J. (1977). Colour vision in the peripheral retina I. Spectral sensitivity. J. Opt. Soc. Am. 67, 195-201.

Anh, S. J., Legge G.E. and Luebker, A. (1995). Printed cards for measuring low-vision reading speed. Vis Res. 35, 1939-1944.

Armstrong, R.A., Eperjesi, F. and Gilmartin B. (2002). The application of analysis of variance (ANOVA) to different experimental designs in optometry. Ophthalmic Physiol. Opt. 22, 248-256. 
Bailey, I., Kelty, K., Pittler. G., Raasch, T. and Roberts G. (1978). Typoscopes and yellow filters for cataract patients. Low Vision Abstr. 4, 2-6.

Bedford, R. E. and Wyszecki, G. (1957). Axial chromatic aberration of the human eye. J. Opt. Soc. Am. 47, 564-565.

Bennett, A. G. and Rabbetts, R. B. (1989). Clinical Visual Optics, $2^{\text {nd }}$ edition. Butterworths, London, UK, pp. 333-334

Bowers, A. R., Meek, C. and Stewart, N. (2001). Illumination and reading performance in age-related macular degeneration. Clin. Exp. Optom. 84, 139147.

Campbell, F.W. and Gubisch, R. W. (1967). The effect of chromatic aberration on visual acuity. J Physiol. 192, 345-358.

Chylack, L. T. Jr, Leske, M. C., Sperduto, R., Khu, P. and McCarthy, D. (1988). Lens Opacities Classification System. Arch. Ophthalmol. 106, 330-334.

Dickinson, C. (1998). Low Vision:Principles and Practice. ButterworthHeinemann, Oxford, UK, p. 35. 
Elliott, D. B., Trukolo-llic, M., Strong, J. G., Pace, R., Plotkin, A. and Bevers, P. (1997). Demographic characteristics of the vision-disabled elderly. Invest. Ophthalmol. Vis. Sci. 38, 2566-2575.

Eperjesi, F., Fowler, C. W., and Evans, B. J. W. (2002). Do tinted lenses or filters improve visual performance in low vision? A review of the literature. Ophthalmic Physiol. Opt. 22, 68-77.

Frennesson, I. C. and Nilsson, U. L. (1993). Contrast sensitivity peripheral to an absolute central scotoma in age-related macular degeneration and the influence of a yellow or an orange filter. Doc. Ophthalmol. 84, 135-144.

Goodrich, G. L., Kirby, J., Keswick, C., Oros, T., Wagstaff, P., Donald, B., Hazan, M. A. and Peters, L. J. (2000). In: Vision '99: Vision rehabilitation: assessment, intervention and outcomes (eds C. Stuen, A. Arditi, A. Horowitz, M.A. Lang, B. Rosenthal, K. Seidman), Swets \& Zeitlinger Publishers b.v., New York, USA, pp. 333-337.

Gormezano, S. and Stelmack, J. (2000). Efficient, effective clinical protocols for the prescription of selective absorption filters. In: Vision '99: Vision rehabilitation: assessment, intervention and outcomes (eds C. Stuen, A. Arditi, A. Horowitz, M.A. Lang, B. Rosenthal, K. Seidman), Swets \& Zeitlinger Publishers b.v., New York, USA, pp. 206-207. 
Hazel, C. A., Petre, K. L., Armstrong, R. A., Benson, M. T. and Frost, N. A. (2000). Visual function and subjective quality of life compared in subjects with acquired macular disease. Invest. Ophthalmol. Vis. Sci. 41, 1309-1315.

Hellinger, G. (1983). The use of CPF lenses for light sensitive individuals. J. Vis. Imp. Blind. 77, 449-468.

Hoeft, W. W. and Hughes, M. K. (1981). A comparative study of low vision patients: their ocular disease and preference for one specific series of light transmission filters. Am. J. Optom. Physiol. Opt. 58, 841-845.

Leat S. J., Fryer, A. and Rumney, N. J. (1994). Outcome of low vision aid provision: the effectiveness of a low vision clinic. Optom. Vis. Sci. 71, 199-206.

Legge, G. E. and Rubin, G. S. (1986). Psychophysics of reading. IV. Wavelength effects in normal and low vision. J Opt Soc Am A. 3, 40-51.

Legge G. E., Ross, J. A., Maxwell, K. T. and Luebker, A. (1989). Psychophysics of reading -VII. Comprehension in normal and low vision. Clin. Vision Sci. $\mathbf{4}$, $51-60$.

Leguire, L. E. and Suh, S. (1993). Effect of light filters on contrast sensitivity function in normal and retinal degeneration subjects. Ophthalmic Physiol. Opt. 13, 124-128. 
Lynch, D. and Brilliant, R. (1984). An evaluation of the Corning CPF550 lens. Optometric Monthly 75, 36-42.

Maino, JH. and McMahon, T.T. (1986). NolRs and low vision. J. Am. Optom. Assoc. 57, 532-535.

Marron, J. A. and Bailey, I. L. (1982). Visual factors and orientation-mobility performance. Am. J. Optom. Physiol. Opt. 59, 413-426.

McClure, M. E., Hart, P. M., Jackson, A. J., Stevenson, M. R. and Chakravarthy, U. (2000). Macular degeneration: do conventional measurements of impaired visual function equate with visual disability? Br. J. Ophthalmol. 84, 244-250.

Morrissette, D. L., Mehr, E. B., Keswick, C. W. and Lee, P. L. (1984). Users' and nonusers' evaluations of the CPF550 lenses. Am. J. Optom. Physiol. Opt. 61, 704-710.

Pensyl, C. D. (1993). Matching patient needs with the features of photochromic filters. Journal of vision rehabilitation 7, 10-12.

Provines, W. F, Harville, B. and Block, M. (1997). Effects of yellow optical filters on contrast sensitivity function of albino patients. J. Am. Optom. Assoc. 68, 353-359. 
Rosenblum, Y. Z., Zak, P. P., Ostrovsky, M. A., Smolyaninova, I. L., Bora, E. V., Dyadina, N. N. and Aliyev, A. G. D. (2000). Spectral filters in low-vision correction. Ophthalmic Physiol. Opt. 20, 335-341.

Tupper, B., Miller, D. and Miller, R. (1985). The effect of a $550 \mathrm{~nm}$ cut-off filter on the vision of cataract patients. Ann. Ophthal. 17, 67-72.

Wilkins, A. J., Nimmo-Smith, I. and Jansons, J. (1992). Colorimeter for the intuitive manipulations of hue and saturation and its role in the study of perceptual distortion. Ophthalmic Physiol. Opt. 12, 381-385.

Wilkins, A. J. (1993). A system for precision ophthalmic tinting: a manual for the Intuitive Colorimeter and trial lenses. Cerium Visual Technologies, Tenterden, Kent, UK.

Wilkins, A. J., Jeanes, R. J., Pumfrey, P.D. and Laskier M. (1996). Speed of Reading Test: its reliability, and its validity in the assessment of the effects of coloured overlays. Ophthalmic Physiol. Opt. 16, 491-497.

Wolffsohn, J. S., Dinardo, C. and Vingrys, A. J. (2002). Benefit of coloured lenses for age-related macular degeneration. Ophthalmic Physiol. Opt. 22, 300311.

Zigman, S. (1990). Vision enhancement using a short wavelength light absorbing filter. Optom. Vis. Sci. 67, 100-104. 
Table 1 Global scale descriptors for the UK version of the Sixteen Personality Factor Questionnaire, 5th Edition

\begin{tabular}{|l|l|l|}
\hline Factor & $\begin{array}{l}\text { Low sten score 1 } \\
\text { to 3 }\end{array}$ & $\begin{array}{l}\text { High sten score 8 } \\
\text { to 10 }\end{array}$ \\
\hline Extraversion & $\begin{array}{l}\text { Introverted, } \\
\text { socially inhibited }\end{array}$ & $\begin{array}{l}\text { Extraverted, } \\
\text { socially } \\
\text { participating }\end{array}$ \\
\hline Anxiety & $\begin{array}{l}\text { Low anxiety, } \\
\text { unperturbed }\end{array}$ & $\begin{array}{l}\text { High anxiety, } \\
\text { perturbable }\end{array}$ \\
\hline $\begin{array}{l}\text { Tough- } \\
\text { mindedness }\end{array}$ & $\begin{array}{l}\text { Receptive, open- } \\
\text { minded }\end{array}$ & $\begin{array}{l}\text { Tough-minded, } \\
\text { resolute }\end{array}$ \\
\hline Independence & $\begin{array}{l}\text { Accommodating, } \\
\text { agreeable, } \\
\text { selfless }\end{array}$ & $\begin{array}{l}\text { Independent, } \\
\text { persuasive, wilful }\end{array}$ \\
\hline Self-control & $\begin{array}{l}\text { Unrestrained, } \\
\text { follows urges }\end{array}$ & $\begin{array}{l}\text { Self-controlled, } \\
\text { inhibits urges }\end{array}$ \\
\hline
\end{tabular}


Table 2 Descriptive statistics for Sixteen Personality Factor Questionnaire, 5th Edition results for the no-tint group

\begin{tabular}{|c|c|c|c|c|c|}
\hline & $\begin{array}{c}\text { Extroversio } \\
\mathrm{n}\end{array}$ & Anxiety & $\begin{array}{c}\text { Tough- } \\
\text { mindedness }\end{array}$ & $\begin{array}{c}\text { Independenc } \\
\mathrm{e}\end{array}$ & Self-control \\
\hline Mean score & 5.0 & 5.2 & 5.2 & 5.1 & 5.3 \\
\hline SD & 1.8 & 2.0 & 1.6 & 1.9 & 1.7 \\
\hline Min. & 0.9 & 0.7 & 1.5 & 1.0 & 0.4 \\
\hline Max & 9.1 & 9.6 & 9.6 & 8.5 & 8.2 \\
\hline $\begin{array}{c}\text { No. of subjects with low } \\
\text { score }\end{array}$ & 16 & 16 & 11 & 23 & 17 \\
\hline $\begin{array}{c}\text { No. of subjects with high } \\
\text { score }\end{array}$ & 11 & 13 & 10 & 12 & 10 \\
\hline
\end{tabular}

Table 3 Descriptive statistics for Sixteen Personality Factor Questionnaire, 5th Edition results for the tint group

\begin{tabular}{|c|c|c|c|c|c|}
\hline & $\begin{array}{c}\text { Extroversio } \\
\mathrm{n}\end{array}$ & Anxiety & $\begin{array}{c}\text { Tough- } \\
\text { mindedness }\end{array}$ & $\begin{array}{c}\text { Independenc } \\
\mathrm{e}\end{array}$ & Self-control \\
\hline Mean score & 5.5 & 5.4 & 5.2 & 4.9 & 5.4 \\
\hline SD & 1.4 & 1.6 & 1.6 & 1.4 & 1.5 \\
\hline Min. & 3.5 & 1.4 & 0.7 & 1.5 & 3.3 \\
\hline Max. & 8.5 & 7.7 & 7.5 & 7.2 & 8.4 \\
\hline $\begin{array}{c}\text { No. of subjects with low } \\
\text { score }\end{array}$ & 2 & 3 & 3 & 2 & 4 \\
\hline $\begin{array}{c}\text { No. of subjects with high } \\
\text { score }\end{array}$ & 3 & 3 & 1 & 1 & 3 \\
\hline
\end{tabular}

